\title{
Germanica
}

\section{Unreliable narrator - programmatische Denkfigur in Felicitas Hoppes Roman Johanna (2006)}

Unreliable narrator - figure programmatique dans le roman Johanna (2006)

de Felicitas Hoppe

Unreliable Narrator in Felicitas Hoppe's Novel Johanna (2006)

\section{Monika Wolting}

\section{OpenEdition}

\section{Journals}

Édition électronique

URL : http://journals.openedition.org/germanica/2657

DOI : 10.4000/germanica.2657

ISSN : 2107-0784

Éditeur

Université de Lille

\section{Édition imprimée}

Date de publication : 30 décembre 2014

Pagination : 13-27

ISBN : 9782913857346

ISSN : 0984-2632

\section{Référence électronique}

Monika Wolting, «Unreliable narrator - programmatische Denkfigur in Felicitas Hoppes Roman Johanna (2006) », Germanica [Online], 55 | 2014, Online erschienen am: 30 Dezember 2016, abgerufen am 06 Oktober 2020. URL : http://journals.openedition.org/germanica/2657 ; DOI : https://doi.org/ $10.4000 /$ germanica. 2657 


\title{
Unreliable narrator - programmatische Denkfigur in Felicitas Hoppes Roman Johanna (2006)
}

\author{
Monika WoLTING \\ Uniwersytet Wrocławski
}

Seit den 60er Jahren werden in der Literaturwissenschaft Diskussionen darüber geführt, warum der Leser die Erzählinstanzen bestimmter literarischer Texte als unzuverlässig, unglaubwürdig, vertrauensverletzend erfährt. Das Empfinden von Unglaubwürdigkeit der erzählenden Entität tritt im Hinblick auf die Darstellungen der Ereignisse in der erzählten Welt oder auch in Bezug auf die subjektive Einschätzung und Bewertung dieser Ereignisse hervor. Felicitas Hoppe schrieb im Jahre 2006 einen Roman unter dem Titel Johanna, in dem die Geschichte der historischen Gestalt Jeanne d'Arc noch einmal dem Leser erzählerisch näher gebracht wird ${ }^{1}$. Es handelt sich dabei um eine fiktionalisierte Geschichte der historischen Figur, die mit viel Einbildungskraft

1. - Es gibt viele Versuche der literarischen Bearbeitung der Geschichte Jeanne d'Arc, zu den Bedeutendsten für die Literaturwissenschaft zählen beispielsweise: Friedrich Schiller: Die Jungfrau von Orleans (1801) ; Bernhard Shaw: Saint Joan (1923) ; Bertolt Brecht: Die heilige Johanna der Schlachthöfe (entstanden 1929/ 39, UA 1959); Jean Anouilh: L'Alouette (1953); Anna Seghers: Prozess der Jeanne d'Arc zu Rouen 1431 (1937). 
und intellektuellem Engagement, der sich darin äußert, das Gegebene infrage zu stellen, verfasst wurde.

In einer nicht näher bestimmten Gegenwart macht sich eine junge Erzählerin - eine Doktorandin - Gedanken über die historische Figur Jeanne d'Arc. Die Icherzählerin führt zahlreiche Gespräche über die Geschichte der französischen Nationalheldin mit ihrem Kollegen, der bereits promoviert und an der Forschung zu Jeanne d'Arc maßgeblich beteiligt ist und ihrem Doktorvater - „Professor“, der als anerkannter Forscher für französische Geschichte gilt. Das ganze Geschehen, wie auch die Gespräche werden aus der subjektiv aufgebauten Position der Icherzählerin geschildert. Der Rezipient wird sich in diesem Kontext die Frage stellen: Welche Funktion soll in einem literarischen Text einer solchen Erzählinstanz zugeschrieben werden? Wenn man davon ausgeht, dass das von der Autorin gewählte Schreibverfahren nicht nur als Eigenschaft eines Textes, sondern als Interpretationsstrategie des Rezipienten gehandelt werden soll, dann ergibt sich daraus die Schlussfolgerung, dass es beim Einsetzen der bestimmten Erzählerfigur um die Vermittlung einer Botschaft gehen könnte. Anhand der im Roman geführten Dialoge werden Streitpunkte des wissenschaftlichen Arbeitens, der Gültigkeit der historischen Abhandlungen und der Bedeutung historischer Denkverfahren hervorgebracht und zur Diskussion gestellt. Die Handlung des Romans findet an unterschiedlichen Orten, die mit der Geschichte Jeanne d'Arcs oder mit der historischen Forschungsarbeit zu tun haben - Hörsaal der Universität, Arbeitszimmer, historische Erinnerungsorte - statt. Die Icherzählerin versucht durch das Geschichtsstudium, die Gespräche mit ihren Kollegen, eigenes Nachdenken und die eigene Vorstellungskraft an die historische, zum Teil zum Mythos gewordene Gestalt näher zu rücken und ihre Geschichte für sich und für den Leser (neu) zu erzählen. Dafür entwickelt sie ihren eigenen Erzählmodus, der sich hauptsächlich aus Fragen, Vergleichen, Vermutungen und Erzählen von Träumen zusammensetzt. Daraus resultiert erstmals eine vorläufige These, deren Bestätigung dieser Beitrag gewidmet wird, dass es sich bei der Icherzählerin im Roman Johanna von Felicitas Hoppe um einen unreliable narrator - einen unzuverlässigen, unglaubwürdigen, unsicheren Erzähler handeln wird. Aus diesem Grund geht es hier um ein pragmatisches Phänomen, dass nicht allein textintern zu beleuchten ist, sondern auch die interpretatorischen Fähigkeiten des Rezipienten mit einschließt. Die Anschlüsse für die Interpretation ergeben sich aus der Differenz zwischen den Absichten der Erzählinstanz und den Normen und auch dem Wissen eines realen Lesers, nicht des impliziten, des vom Autor mitgedachten Lesers. Nünning betont: „Hat der Leser die mangelnde Zuverlässigkeit des Erzählers anhand bestimmter textueller Signale einmal durchschaut, dann erhalten aufgrund dieses 
Informationsvorsprungs die Aussagen des Erzählers eine diesem nicht bewusste und von ihm nicht beabsichtigte Zusatzbedeutung“"2. Dieser Beitrag geht in erster Linie der Aufdeckung der textinternen Signale für die Ermittlung des unreliable narrators und im zweiten Schritt der Bedeutung derer für die Interpretation des Textes nach.

Das erste Anliegen des Beitrags ist die Darstellung und Charakterisierung der narrativen Instanz im Roman Johanna von Felicitas Hoppe. Unter Rückgriff auf theoretische Ausführungen zum Erzähler werden narrative Ebenen und Funktionen des Erzählers bestimmt. In einem weiteren Schritt wird in Anlehnung an Überlegungen von Ansgar Nünning die Icherzählerin des Romans als Beispiel eines unzuverlässigen/unglaubwürdigen Erzählers herausgestellt. Das zweite Anliegen des Beitrags ist, die Ergebnisse der vorgenommenen Untersuchung in Korrelation mit Hoppes literarischer Skepsis der aufklärerischen Wahrheit, der Geschichtsschreibung und der Schrift gegenüber zu setzten. Die These für diese Ausführungen lautet: Felicitas Hoppe kreiert einen unreliable narrator mit dem Zweck, ihre literarische Skepsis dem Erzählen gegenüber nicht nur auf der Inhalts-, sondern auch der Formebene darzustellen.

\section{Darstellung der narrativen Instanz}

Die Icherzählerin kreiert mit ihrer Geschichte eine neue Weltvorstellung, ihre eigene Weltvorstellung. Das Schreiben von Felicitas Hoppe fügt sich in die Tradition der Dichtung ein, die sich indirekt und exemplarisch auf die Welt bezieht, die dem Leser quasi die Welt vorführt und ihm ein Modell der Welt anbietet. Wenn von dieser Prämisse ausgegangen wird, dann wird die Frage nach der Erzählsituation in Johanna von Felicitas Hoppe legitimiert sein. Wie wurde die Erzählerinstanz in dem Roman konstruiert? Was sind ihre Haupteigenschaften? Und wozu sollen die Merkmale der Entität bei der Entwicklung der Handlung und der Entstehung von Figuren dienen? Unter Rückgriff auf narratologische Kategorien wird nachfolgend den Fragen „Wer spricht?“ und „In welcher Situation spricht er/sie?“ nachgegangen. Hoppes Erzähler wird als Teil der erzählten Geschichte kreiert. Diese Geschichte wird dem Rezipienten von der Erzählerinstanz aus ihrer eigenen Sicht erzählt. Dabei handelt es sich um eine weibliche Erzählerin, eine Doktorandin des Fachs Geschichte, die sich auf das Rigorosum, in dem sie zu Geschichte Jeanne d'Arc sprechen soll, vorbe-

2. - Ansgar Nünning: „Unreliable Narration zur Einführung. Grundzüge einer kognitiv-narratologischen Theorie und Analyse unglaubwürdigen Erzählens", in: Ansgar Nünning: Unreliable Narration. Studien zur Theorie und Praxis unglaubwürdigen Erzählens in der englischsprachigen Erzählliteratur, Trier, Wissenschaftlicher Verlag Trier, 1998, S. 17. 
reitet und das Rigorosum absolviert, allerdings erfährt der Leser das Ergebnis der Prüfung nicht.

Die abwechselnd verwendeten Zeitformen - Präteritum und Präsens - deuten auf die Vorrangigkeit der erzählten Geschichte gegenüber der Narration.

Er SCHNAPPTE nach Luft, HOB sein Glas, und ich LACHTE, weil Peitsche kein Taucher IST, sondern Schwimmer, das SIEHT man gleich. [...] Aber er PROSTETE mir entschlossen zu, wobei er sogar ein wenig SCHWANKTE, obwohl er gar nicht betrunken WAR. Denn im Gegensatz zu mir TRINKT Peitsche fast nie [...] Geliebter Gegner, er DREHT mir den Strick $(\mathrm{J}, 15)^{3}$. (Hervorhebung: MW)

Die Erzählerin berichtet über das Gewesene und fügt Kommentare, Erklärungen, ihre Überlegungen in der Jetzt-Form ein. Dadurch wirkt ihre Erzählung nicht abgeschlossen, sie ist ständig modellierbar und ununterbrochen neu interpretierbar. Durch diese Berichterstattungsform wird dem Rezipienten die Modalität und Unzuverlässigkeit der Erzählung aus der Vergangenheit signalisiert.

Die Erzählerin nimmt im Roman die Position der Hauptfigur ein. Über die Titelfigur - Johanna - wird im Roman lediglich berichtet oder sie wird rhetorisch angesprochen. Dieses Verfahren eröffnet der Autorin die Möglichkeit zur Diskussion über Fragen der Geschichte, des Mythos oder der Historiografie.

Die Figur der Icherzählerin kennzeichnet sich durch ihre fortwährende Präsenz in der Diegese (im Sinne von Genette), die von ihr gänzlich gestaltet und bestimmt wird. Keiner anderen Figur wird der Raum gewährt, die eigene Sicht der Ereignisse darzustellen. Die Icherzählerin schafft ein Universum ihrer eigenen Erzählung. Was der Rezipient erfährt, steht im direkten Zusammenhang mit der Erzählinstanz, große Textpassagen werden in dialogischer Form verfasst, die Dialoge werden allerdings in den inneren Monolog der Icherzählerin eingebunden, ohne dass Anführungsstriche oder andere sprachliche Markierungen für die Kennzeichnung der Rede angewendet werden.

Und Sie, fragte ich. Für wen halten Sie sie? Er sah mich an, ließ die Hände sinken, ging kurz in die Knie und griff nach dem Glas. Dann erhob er sich wieder. Ich halte mich an das, was ich anfassen kann, sagte er. Übrigens führt diese Frage zu nichts, weil sie den Kern der Sache verfehlt. Die Vergangenheit interessiert mich nicht, ich glaube an das, was ich sehe und höre. [...] Er lehnte im Rahmen der offenen Küchentür, er sprach leise und schnell, wie ein ungeübter talentloser

3. - Felicitas Hoppe: Johanna. Roman, Frankfurt am Main, S. Fischer Verlag, 2006. Im Text mit dem Kürzel (J) gekennzeichnet, die Seitenangaben folgen dem Kürzel $\mathrm{J}$. 
Lügner, der nicht weiß, wie man Wahrheit verbirgt und dass man beim Lügen ernsthaft sein muss (J, 16-17).

Die Erzählerin agiert auf zwei Ebenen, auf der Ebene der erzählten Geschichte und auf der Ebene der Narration. Sie erzählt auf der Ebene der Geschichte von vergangenen Ereignissen, die eng mit den Vorbereitungen auf das Rigorosum zusammenhängen. Auf der Ebene der Narration fügt sie der Erzählung der ersten Ebene Kommentare, Reflexionen, Analysen, Beschreibungen der eigenen Empfindungen und Wiedergaben der Gedanken aus dem Jetzt der Erzählsituation zu.

Die weiteren Figuren des Romans: Professor, Peitsche (Doktor), Jeanne d'Arc werden aus der Sichtweise der Erzählerin dargestellt und hauptsächlich durch Vergleiche mit der Erzählerin konstruiert. Das Bild der Erzählerin ergibt sich aber auch aus dem Vergleich mit den anderen Romanfiguren, durch eingesetzte Verneinungen oder durch eine festgestellte Ähnlichkeit.

Doktor Peitsche, mein Vorbild. Schnelle Zunge, helle Stimme, schlagende Rede, biegsamer Gang (J, 11). [...] wovon Johanna nichts weiß und was mich so gründlich mit Peitsche verbindet: Feigheit, Gewohnheit, Manieren, der Wunsch nach der besseren Hälfte des Mantels (J, 13). Jedenfalls bis der Professor den Schauplatz betritt [...] der im Gehen die maigrüne Mütze schwenkt, mit der Linken, versteht sich. Und sofort werde ich mich zu schämen beginnen, weil ich selbst natürlich kein Linkshänder bin (J, 98).

Das unklare, in Details zerlegte Bild der Erzählerin wird während der Lektüre noch durch ständiges Infragestellen des Selbst und der erzählten Geschichte konturenloser.

Aber bin ich mir sicher? Stehe ich wirklich im Licht? Für wen halte ich mich? Was will ich entziffern (J, 22)?

Dieser Umstand führt zu der Überlegung, ob es sich bei der Kreation der narrativen Instanz um einen Typus des unsicheren Erzählers handelt? Falls sich dieser Gedanke bestätigen sollte, ist davon auszugehen, dass Felicitas Hoppe auf zweifache Weise sich der Exemplarität der vorgeführten Welt, einem Modell der Wirklichkeit, annähert. Zum einen geschieht es durch die dem Text auf der Inhaltsebene eingeschriebene Skepsis der historischen Wahrheit gegenüber und zum anderen durch die Auswahl des Modells des unglaubwürdigen/ unsicheren Erzählers.

Durch die Auswahl dieser Erzählinstanz bestätigt die Autorin umso deutlicher den Gedanken, den die Wissenschaft schon längst zum Kern ihrer Auseinandersetzung gemacht hat. Die wissenschaftliche Skepsis und Reflexion über diese Themenkomplexe fand in die wissenschaftli- 
chen Abhandlungen unterschiedlichster Provenienz, beispielsweise von Jean-François Lyotard, Reinhart Koselleck, Jean Baudrillard, Vilém Flusser, Hayden White oder Jacques Le Goff Eingang 4 . So konstruiert auch Felicitas Hoppe ihre Figuren auf den Ebenen unterschiedlicher Geschichtsauffassung. Die Autorin geht in ihrem Text Johanna der Frage der Diskursivität eines der größten Nationalmythen Frankreichs - der Geschichte von Jeanne d'Arc - nach.

Daraus ergibt sich eine weitere gewichtige Frage nach der Funktion von Hoppes Literatur. Die Autorin greift das Thema der postmodernen Haltung der Geschichtsschreibung gegenüber auf und realisiert dieses im Roman mit den der Literatur zur Verfügung stehenden Mitteln. Es wird von Hoppe ein fragmentarisches, vorläufiges Modell des Umgangs mit der Schrift, mit der Wissenschaft, mit der Gültigkeit von Fremdund Eigenaussagen überhaupt entwickelt. Damit leistet sie einen literarischen Beitrag zu der oben angedeuteten postmodernen Diskussion über den Abschied von großen, sinnstiftenden Metaerzählungen.

\section{Die Icherzählerin als unreliable narrator}

In Johanna lassen sich mehrere textuelle Signale für die Kreation eines unzuverlässigen Erzählers - unreliable narrator festmachen. Ausgehend von Wayne C. Booths klassischer Definition „I have called a narrator reliable when he speaks for or acts in accordance with the norms of the work (which is to say, the implied author's norms), unreliable when he does not" 5 lassen sich folgende Kriterien des unzuverlässigen Erzählers in einem an historische Ereignisse angelehnten Roman feststellen6: 1. Der Icherzähler ist zugleich auch die Hauptfigur des Romans; 2. Ein zweites Kriterium, das von Ansgar Nünning angeführt wird, meint den hohen Grad an Explizität, mit der der Erzähler als konkret fassbarer, personalisierbarer Sprecher auf der Ebene der erzählerischen Vermittlung in Erscheinung tritt; 3. Ein drittes Kennzeichen des unzuverlässigen Erzählens, das auf Hoppes Werk zu übertragen wäre, ist die häufige Verwendung von Kommentaren, interpretatorischen Zusätzen, einer Anhäufung von persönlichen Stellungnahmen

4. - Die Bedeutung der historischen Wissenschaften erfuhr im 20. Jahrhundert eine signifikante Umwertung, sie wurde als objektive Erklärungsinstanz der Welt und des Menschen delegitimiert. Lyotard ist der Auffassung, dass das entscheidende Kriterium in der Durchsetzung eines Gedankens nicht sein Wahrheitsgehalt ist, sondern sein Produzent, bzw. die Macht seines Produzenten ist. Auch Vilém Flusser erörtert in seinen Texten den Konstruktionscharakter der Geschichte und unterzieht ihn seiner Kritik. In: Vgl.: Vilém Flusser: Nachgeschichte. Eine korrigierte Geschichtsschreibung, Bensheim/ Düsseldorf, Bollmann, 1993, S. 25.

5. - Wayne C. Booth: The Rhetoric of Fiction, (1961) University of Chicago Press, 1983, S. 158-159.

6. - a.a.O., Nünning, 1998, S. 6. 
seitens der Erzählerin, sowie auch Leseranreden; und 4. Als charakteristisch für die unsichere, unzuverlässige bzw. unglaubwürdige Narration wird des Weiteren eine Diskrepanz zwischen dem, was ein unreliable narrator dem Leser zu vermitteln versucht und einer anderen Version des Geschehens, die dem fiktiven Adressaten wohl bekannt sein dürfte, erwähnt. Die andere Version des Geschehens wird häufig unbewusst von dem Erzähler an den Leser übermittelt, was letztendlich zu einer Selbstentlarvung des Erzählers führt.

\section{Die Icherzählerin als Hauptfigur des Textes}

Aus den skizzenhaft dargestellten Kriterien für die Ermittlung des unzuverlässigen Erzählers nach Nünning leiten sich für den Roman Johanna folgende Konsequenzen ab: Die Icherzählerin ist die Hauptfigur des Romans, sie berichtet von den Ereignissen, die unmittelbar vor ihrem Rigorosum liegen. Die Beschäftigungen, denen sie an diesen Tagen nachgeht, sind eng mit dem Prozess der Aneignung der historischen Fakten aus dem Leben der historischen Figur Jeanne d'Arc verbunden. Das Auswendiglernen des historischen Stoffes wirft bei der Erzählerin viele Fragen auf, die sie im Laufe des Romans zur Sprache bringt. Da die Erzählerin zur Hauptfigur des Romans wird und zur einzigen Erzählinstanz, lernt der Rezipient die erzählte Welt ausschließlich aus der Perspektive der Erzählerin durch ihre erzählerische Vermittlung kennen. Auch die stattgefundenen Gespräche werden von der Erzählerin wiedergegeben. Sie übernimmt die absolute Gewalt über das Erzählte. Die weiteren Figuren: Peitsche (Doktor), Professor und Johanna werden dem Leser durch das Prisma der Erzählerin dargeboten.

\section{Die explizite Erzählerin}

Der Typus der Erzählerin gehört der Reihe der expliziten Erzähler an, da ihr unzuverlässiges Erzählen die Aufmerksamkeit auf ihre Person lenkt. Die Icherzählerin bestimmt auf der Kommunikationsebene die Handlungen und das Geschehen. Durch ihre Wahrnehmung bietet sie dem Rezipienten auf der Rezeptionsebene eine konkrete Perspektive durch zum größten Teil einen begrenzten Informationsstand und aufgrund ihrer emotionalen Anteilnahme an der erzählten Geschichte an ${ }^{7}$.Es ist also anzunehmen, dass die erzählten Ereignisse aus dem Leben der historischen Figur Jeanne d'Arc verzerrt dargestellt werden könnten, bzw. mit Fragen versehen werden, wo die historische Wissenschaft schon längst meint, alle Antworten gefunden zu haben. Dies wird schon

7. - Ebd., S. 9. 
auf den ersten Seiten des Romans deutlich, auf denen die Icherzählerin die uneindeutige Geschichtsschreibung herausstellt:

Am dreißigsten Mai, gegen neun, flankierten ACHTZIG oder ACHTHUNDERT englische Soldaten ihren Karren auf dem Weg zum Alten Markt von Rouen (J, 9). (Hervorhebung MW)

In dem ersten Kapitel, unter dem Titel „Prolog“ wird der Anschein erweckt, dass ein Erzähler dem Rezipienten eine Zusammenfassung der Geschichte von Jeanne d'Arc darbietet. Die Erzählerin kommuniziert allerdings dem Leser eine eher unklare Geschichtsschreibung, womit sie auf Defizite kognitiv-epistemologischer Natur hinweist. Schon der erste Eröffnungssatz des Romans „Johanna wurde in der Dreikönigsnacht geboren (J, 9)“ deutet auf eine Willkür im erzählerischen Verfahren hin, da das Geburtsdatum am 06. Januar weder historisch bewiesen noch bestritten wird. Allerdings muss es sich bei der Bezeichnung „die Dreikönigsnacht" nicht unbedingt um den 6. Januar handeln, da auch dieses Datum nur normativ festgelegt wurde. An diesem Beispiel wird deutlich, wie die Kommunikationsebene des Romans aufgestellt wird. Der Rezipient wird in dem Roman nicht mit nachforschbaren Fakten konfrontiert, sondern mit den Fragen nach legendärer Überlieferung. Die Überlieferung wirft vom ersten Satz des Romans Zweifel auf; auch die Erzählerin besitzt keine Gewissheit über die historischen Ereignisse. Auf dieser Erzählebene fügt sich Felicitas Hoppes Schreiben in die postmoderne Tradition der Anzweiflung der Geschichtsschreibung überhaupt ein.

Der hohe Grad der Explizität der Erzählweise wird zudem dadurch unterstützt, dass die Erzählerin unaufhörlich über ihre Gedanken, Empfindungen und Überlegungen berichtet und durch die eigene Wiedergabe der geführten Dialoge mit anderen Figuren keine andere Person zum Wort kommen lässt. Sie ist die alleinige Instanz, die die Inhalte und Ereignisse zum Erzählen bereitstellt. Seymour Chatman hat für diese Art der Fokalisierung den Terminus „fehlbarer Filter"8 gestiftet. So lässt sich vermuten, dass einige der berichteten Dialoge zum Teil auch als „fehlbar“ eingestuft werden können, da sie alle aus dem Gedächtnis der Erzählerin wiedergegeben werden und der Rezipient keine Chance auf Revision des Erzählten hat.

Dann die Schlacht von Patay. Eine Schlacht, an die Peitsche sich gerne erinnert. Es gibt sogar Bilder. Und das hier bin ich, sagt Peitsche stolz und schlug eine Seite im Album um, ein Kind unterm Helm. Daneben Johanna, ein Haarschnitt wie eine Puddingschüssel. Unter der

8. - Seymour Chatman: Coming to Terms. The Rhetoric of Narrative in Fiction and Film, Cornell University Press, 1990, S. 149. 
Schüssel ein ernstes Gesicht, vor den Augen ein Ziel. Auf Peitsches Abbildung allerdings etwas verschwommen (J, 84).

Somit rückt nicht die Handlung, sondern die Perspektive der Erzählerinstanz ins Zentrum des Textes. Die Aufmerksamkeit des Rezipienten, wie schon im Punkt 1. ausführlich erörtert wurde, verlagert sich von der Ebene des Geschehens auf die Ebene des reinen Erzählens. Die Verlagerung des Interesses macht es dem Rezipienten die Unterscheidung von fiktionalem und faktualem Erzählen gar unmöglich.

\section{Subjektive Erzählerin}

Die Erzählerin wird mit einem Modus ausgestattet, der sich aus Kommentaren, Gedankensplittern, unüberprüfbaren Situationen, Behauptungen, widersprüchlichen Äußerungen, Verschmelzung der Zeitebenen, unbegründeten Urteilen, Antizipierungen, zynischen Anmerkungen, Wiederholungen von Teilen der Narration in immer neuen Kontexten, Anspielungen, offenen Fragen, Verschmelzen von Traumund Wirklichkeitsebenen, Ausrufen und Leseranreden und -ansprachen zusammenfügt. Im Weiteren soll auf einige dieser Techniken genauer eingegangen werden.

Die rezipientenorientierte Schreibweise wird an der mehrmals im Text verwendeten Formel „Damen und Herren“ deutlich. Durch den Gebrauch von Leseranreden unternimmt die Autorin einen bewussten Versuch der Rezipientenlenkung durch die Erzählerin ${ }^{9}$. Die Erzählerin leitet auf diese Weise mehrere Fragen, die an den Rezipienten gerichtet werden, ein. Die Fragen erweisen sich des Öfteren als rhetorisch gemeint, da die Erzählerin gleich darauf eine Antwort liefert.

Damen und Herren, die Wahrheit, was ist das (J, 23)?

Damen und Herren, was ist das? Eine große gigantische Prahlerei $(\mathrm{J}, 27)$.

Die Anredeformel wird auch in einem Zusammenhang verwendet, in dem eine Gemeinsamkeit zwischen der Icherzählerin und dem Rezipienten gestiftet werden soll.

DAMEN UND HERREN, das war zu erwarten. Also doch eine Hexe $(\mathrm{J}, 86)$.

DAMEN UND HERREN, draußen ist Mai, vergessen wir einfach den kleinen Krieg (J, 93).

DAMEN UND HERREN, nur damit Sie es wissen (J, 97).

9. - Vgl., a.a.O., Nünning, 1998, S. 28. 
DAMEN UND HERREN, wir stellen uns einfach vor, neunzehn zu sein, dann wird es schon gehen (J, 140).

Aber DAMEN UND HERREN, für wen immer gebaut wird, Geduld ist gefragt, denn der Bau finanziert sich durch Steuer, die uns fasten erlaubt (J, 160). (Hervorhebung: MW)

Der Leser wird im Roman direkt angesprochen, er wird darauf verwiesen, sich Gedanken zu der von der Erzählerin aufgeworfenen Problematik zu machen. Nicht selten spricht die Erzählerin in der Form „wir“", womit sie sowohl den Rezipienten als auch ihre eigene Entität meint:

So kommen WIR niemals über die Schwelle (J, 28).

WIR bleiben zurück und schwimmen in Tümpel der Angst vor uns selbst (J, 37).

Stattdessen können WIR kochen und spinnen und heimlich häusliche Feuer schüren [...] (J, 39).

Wie klein WIR sind, wenn der Morgen anbricht, der UNS mit einem Licht versorgt, das alles erbärmlich ins Deutliche rückt (J, 43). (Hervorhebung: MW)

Die Erzählinstanz wendet sich zudem auch eindeutlich an Johanna selbst und auch an ihren Kollegen, Freund, Bekannten - Peitsche.

Geliebte Johanna (J, 87); Willkommen Johanna (J, 88); A hnungslose Johanna (J, 94) !; Geliebter Gegner (J, 120) !

Auch an den Rezipienten werden Fragen gerichtet, deren Beantwortung für ein besseres Verständnis von Johannas Persönlichkeit und Haltung Bedeutung haben könnte. „Übrigens, konnte Johanna schwimmen (J, 26)?“ Im Fortlauf des Textes wird die Frage wiederholt und zugleich beantwortet: „Übrigens, konnte Johanna schwimmen? Johanna konnte sicher nicht schwimmen (J, 51).“ Auch die Frage „Ob Johanna gesprochen hat" $(\mathrm{J}, 44)$ ist zum Teil auch rhetorisch gemeint, da auch hier die Antwort an die Frage angehängt wird ,Natürlich hat Johanna gesprochen (J, 44).“

Die Fragen der Erzählerin gestalten sich als Fragen sehr unterschiedlicher Provenienz. Eine Art davon soll hier herausgestellt werden, da sie unmittelbar die intellektuellen Fragestellungen nach der Historie, der historischen Überlieferung, dem geschichtswissenschaftlichen Arbeiten, wie auch auf der zweiten Ebene der eigenen Poetik der Autorin Felicitas Hoppe betreffen.

Worauf kommt es in der Geschichte an? Nicht darauf, dass man Geschichte erzählt, sondern, wie man Geschichte macht, wenn man erzählt (J, 47). 
Damit schafft die Autorin zum einen eine direkte Anknüpfung an die Fragen der Philosophen, Historiker und Soziologen wie JeanFrançois Lyotard, Jaques Le Goff, Vilém Flusser oder Hayden White. Zum anderen macht sie den Leser auf die Glaubwürdigkeit, oder auch Unglaubwürdigkeit der Erzählung der Icherzählerin aufmerksam. An diesem Satz könnten die Aspekte der Verlässlichkeit, Glaubwürdigkeit und Wahrhaftigkeit ${ }^{10}$ eines Erzählers festgemacht werden.

Die Annahme oder Übernahme Jeanne d'Arcs zugeschriebenen Redewendungen und Ausrufe, die immer wieder in neue Kontexte eingefügt werden, stellt einen weiteren Beweis für die Formel einer vertrauensunwürdigen Erzählinstanz dar. Der Ausruf „Heilige Einfalt““ wird mehr als 10 Mal im Texte eingeführt (J, 18, 37, 46, 77, 87, 94, 141, 143, 170), einmal kommt er in einer veränderten Form „R Retorische Einfalt (J, 101).“ vor. Auch die Wendungen „Par mon Báton, par mon Martin (J, 39, 53,55, 68, 110, 142, 154)“ und „Jesus Maria (J, 57, 58, 105,

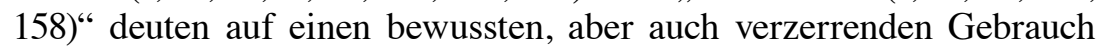
von historisch überlieferten Materialien.

Auf der Erzählebene verschmelzen zum Teil die historische Zeit der Geschichte Jeanne d'Arcs mit der Erzählzeit zu einer unbestimmten Zeitebene:

Johanna brennt und ich sitze im Saal (J, 34).

Johanna brennt, und ich schlafe (J, 38).

Ich liege im Bett, und die Jungfrau gewinnt ihre erste Schlacht (J, 39).

Sie zu den Soldaten, wir ab ins Schwimmbad, sie in den Krieg und wir in den Sommer (J, 51).

Sie in die Schlacht und wir ins Vergnügen (J, 51).

Ich schlafe und Johanna verliert die Schlacht $(\mathrm{J}, 86)$.

Johanna brennt und ich schwimme (J, 169).

Johanna widerruft, und ich singe (J, 143).

Aber wo ist Johanna? Wo ist Peitsche (J, 149)?

\section{Diskrepanz}

Das vierte Phänomen, das von Nünning angesprochen wurde, ist die „Diskrepanz zwischen dem, was ein unreliable narrator dem fiktiven Adressaten zu vermitteln versucht und einer zweiten Version des Geschehens, [...] die sich dem Rezipienten durch implizite Zusatzinformationen erschließen kann"11. Die Icherzählerin vermittelt dem Leser ihre eigene Sicht auf die historischen Ereignisse, Fakten und Personen. $\mathrm{Zu}$ einer Konfrontation ihrer Auffassung der Geschichte

10. - Vgl.: Franz Karl Stanzel: Theorie des Erzählens, Göttingen, UTB, 1979, S. 201-202.

11. - a.a.O., Nünning, 1998, S. 6. 
mit einer anderen Auslegung kommt es in der Prüfungssituation, als der Professor einen anderen Wissensstand präsentiert und von der Doktorandin erwartet. Damit überführt sich die Icherzählerin selber. Sie stellt Behauptungen auf, wo die Wissenschaft im Unklaren bleibt, gleichzeitig wirft sie da Fragen auf, wo die Wissenschaft meint, die Antworten längst gefunden zu haben. Felicitas Hoppe entzieht sich der Pflicht über die einzig richtige geschichtliche Wahrheit zu entscheiden und lässt das Ergebnis der Prüfung aus. Damit bezweifelt sie umso mehr die Möglichkeit einer Annäherung an die Wahrheit der Geschichte.

\section{Literarische Skepsis der Geschichtsschreibung gegenüber}

Damit komme ich zum letzten Punkt meiner Auseinandersetzung mit dem Thema des unreliable narrators als einer programmatischen Denkfigur im Werk Hoppes. Hoppe liefert mit ihrem Text die literarische Skepsis der aufklärerischen Wahrheit, der Geschichtsschreibung und der Schrift gegenüber. Die Autorin schuf einen unreliable narrator mit dem Zweck, ihr intellektuelles Anliegen nicht nur auf der Inhaltsaber auch der Formebene vor den Rezipienten zu bringen.

Hoppes Figuren werden mit Funktionen und Eigenschaften unterschiedlicher Historiker ausgestattet, damit stehen sie für verschiedene Herangehensweisen an historische Stoffe. Peitsche und Professor haben klare, wenn auch unterschiedliche Vorstellungen, wie Geschichte funktioniert und wie sie an nachfolgende Generationen zu vermitteln ist. Die Icherzählerin stellt ihre Vorgehensweise in Frage, sie versucht eine subjektive, persönliche, emotionale Annäherung an den zu untersuchenden Gegenstand. Diese Prämissen haben den amerikanischen Historiker Heyden V. White beschäftigt und ihn zu der These verleitet: „Auch Klio dichtet". White ging in seinem Werk Metahistory ${ }^{12}$ davon aus, dass jede Darstellung historischer Ereignisse poetologischen Kategorien unterliegt. Die Autorin scheint diesen Gedanken aufzugreifen und lässt die Figuren ihres Textes danach vorgehen. Sie alle versuchen, eine eigene Geschichte zu denken, zu schreiben und zu ,falten“, die unter Umständen zu einer allgemeingültigen Geschichtsauffassung werden könnte. Der Typus des unglaubwürdigen Erzählers bestätigt nur noch deutlicher die Unmöglichkeit, sich an geschichtliche Fakten zu nähern, die Geschichte wird immer aus verschiedenen Facetten konstruiert, sie bleibt immer eine gedichtete Geschichte. Das kulturelle Gedächtnis, das in Museen, Geschichtsbüchern, Archiven, „Mützenzimmern“ aufbewahrt wird,

12. - Heyden V. White: Metahistory: The Historicar Imagination in Nineteenth century / Metahistory, die historische Einbildungskraft im 19. Jahrhundert in Europa. Frankfurt am Main: Fischer 1991. 
wird mit poetologischen Mitteln geschrieben und demzufolge befindet es sich in einer ständigen Umformulierung, Neugestaltung, nicht selten auch Anpassung an die Erwartungen der es rezipierenden Generation.

Die von der Wissenschaft gewählte Methode einer Annäherung an Erinnerung kann nicht erfolgreich werden, da der Wissenschaftler für die Wahrheit über eine historische Gestalt andere Aufschreibsysteme benötigte als diejenigen, die der Wissenschaft zu Verfügung stehen. Die Icherzählerin sucht nach anderen Wegen, Johanna näher zu kommen, sie unternimmt das Wagnis, sich ihr nicht durch die Schrift, sondern durch die Sprache zu nähern. Sie schafft durch ihr Nachdenken eine Art mündlicher Kommunikation mit Johanna, auch wenn die historische Gestalt nicht antwortet, so kommt die Icherzählerin zumindest in einen Dialog mit dem Leser über ihre Gestalt. Der Leser, der reale Leser oder im Ecoschen Sinne - der Modellleser -, der die Leerstellen des Textes mit seinem auch enzyklopädischen Wissen zu füllen neigt, hat die Chance sich während der Lektüre zu einem mit-fragenden und mit-denkenden Leser zu entwickeln. Die Qualität der archivierten Erinnerung reicht der Icherzählerin im Gegenteil zu Peitsche und zum Professor nicht aus, sie bringt die Vergangenheit in ihre eigene Erfahrung. Sie wählt eine eigene Methode für diese Herangehensweise, sie basiert nicht auf Dokumentensichtung, auf einem Besuch der Erinnerungsorte, sondern auf reinem Nachdenken, Abwägen, Überlegen, Situationen Durchspielen, auf Sich-in-die-Gestalt-Hineinversetzen. Ihre Auseinandersetzung entspringt dem Widerstand gegen die Ablösung von lebendiger Geschichtserfahrung durch wissenschaftliche Forschung, die von Peitsche betrieben wird und vom Professor verlangt wird. Das wissenschaftliche Wissen, das in Hoppes Text mit dem auswendig gelernten Wissen gleich gesetzt wird, wird von der Icherzählerin mit Widerwillen von sich gewiesen. Das Interesse der Erzählerin gilt grundsätzlich anderen Begebenheiten als es dem allgemeinen, enzyklopädischen, durch Wissenschaft kontrollierten Wissen zu entnehmen ist:

Morgen habe ich Prüfung, und der Professor wird mich ganz sicher NICHT (Hervorhebung, MW) fragen, von welcher Seite die Sonne schien, als jemand die grüne Mütze schwenkte. Er wird mich nach etwas anderem fragen, vermutlich nach den zwölf Schuldartikeln, die ich noch immer nicht auswendig kann (J, 103).

Ihr selbstständiges Nachdenken wird alsbald in der Prüfungssituation als „Romane schreiben“ bezeichnet:

Liebe Dame! Wissen Sie, wie das die Wissenschaft nennt? Sie sehen nicht so aus, als ob Sie das wissen [...]. Tatsächlich, Sie sollten Romane schreiben, mir scheint, Sie haben das Zeug dazu, Einbildungsund Empfindungskraft. Sinn für die Szene, für Pomp und Posaune. 
Für Reiter und Ross. Das so genannte Naturelement, eine schöne und blühende Phantasie. Sinn für Farben und Stimmen. Für die richtige Stimmung, für Atmosphäre. [...] Sie lieben das Einfache, kann das sein $(\mathrm{J}, 123 \mathrm{f})$ ?

Das Fach Geschichte, das die Inhalte aus dem Archiv bezieht, ist kein Ort für „Einbildungskraft“ und „Empfindungskraft“, für „,blühende Phantasie“, für den „Sinn für Farben und Stimmen“, sondern für wissenschaftliches Arbeiten an den Quellen und Dokumenten.

„In der Wissenschaft geht es um Aufmerksamkeit (J, 74), Eine Übung in Eleganz und Geduld. Zögern adelt, ein Zeichen von Klasse, eine höhere Form von Präzision [...] ERST DENKEN, DANN HANDELN, das ist die Devise“ (J, 32, 40).

Dieses Arbeiten setzt die Icherzählerin jedoch gleich mit Umschreiben, Verfälschen, Kassieren vom scheinbar Unnötigen, Zusammenstellen vom Zusammenhangslosem. Die Historiker sind für sie „falsche Priester“ (J, 14), „Wächter in Waffen, die nichts zu verteidigen haben, nur Thesen und Mützen“ (J, 14). Das Interesse der Icherzählerin gilt der Beschreibung und dem Wahrnehmen von Gerüchen, Atmosphären, Farben, Temperatur, Geräuschen, „einer Ahnung von Frischluft, einem Hauchs von Idee“" (J, 57), den Gefühlen einer zur Scheiterhaufen Geführten:

Denn morgens zwischen neun und halb zehn ist das Leben noch kurz, keine Zeit für ein Frühstück, nicht mehr als drei Schritte zum Scheiterhaufen. Danach eine Predigt, ein Stoßgebet, ein eilig hingekritzeltes Zeichen, kürzer als jede Unterschrift. Der Rest geht in einer Ewigkeit auf, für den auf dem Markt von Rouen kein Platz ist (J, 34).

In dem Erproben, Nachfühlen, Nachdenken, in dem dialogischen Vorgehen mit den Texten des kulturellen Gedächtnisses erarbeitet die Icherzählerin für sich eine Arbeitsmethode für den Umgang mit der historischen Figur Johannas. Dies wird noch einmal deutlich im letzten Kapitel des Romans. Hoppe lässt ihre Figuren durch das Jeanne d'ArcMuseum in Rouen wandern, wo historisch überlieferte Stationen aus dem Leben Johannas in Wachs verewigt worden sind. Wachs - ein Stoff, der bei der kleinsten Wärmezufuhr zu einer Wachslache wird, genauso wie Papiermützen im Kontakt mit Feuer zu Asche und mit Wasser zu einer klumpigen Masse werden. Nach dem Museumsbesuch, an dem auch Kinder teilnehmen, schlägt der Museumsführer, dessen Dienste in der postmodernen Welt nicht mehr erwünscht sind, vor:

Kinder, reißt euch die Mützen vom Kopf, und zeigt mir die Ohren, damit wir hören, was sonst keiner hört, alle Stimmen, die für Johanna 
sprechen! Was übrigens die Führung betrifft, damit sind wir am Ende, was dagegen Johanna betrifft, so fängt die Geschichte erst an. Und zwar jetzt und gleich hier (J, 164).

Der Roman stellt einen ästhetischen Ausdruck einer Notwendigkeit dar, die vorherrschenden historischen Diskurse, die traditionellen Formen der Memorierung kritisch zu beleuchten. Felicitas Hoppe schreibt mit ihrem Roman einen gewichtigen literarischen Beitrag zu der intellektuellen Debatte über das kulturelle Gedächtnis, über die Zukunft der Schrift und der mit ihr verbundenen Aufschreibsysteme. Mit der Figur der Icherzählerin, die als unzuverlässiger Erzähler einzustufen ist, schafft die Autorin eine junge Intellektuelle, die das wissenschaftliche Arbeiten vorführt, am eigenen Beispiel die Mechanismen der Geschichtsschreibung aufzeigt, das Funktionieren der Aufschreibsysteme überprüft, den Fragen nach der Bedeutung von Historie für die Zukunft, der Konservierung von Vergangenheit und den Rahmenbedingungen der historischen Wissenschaften nachgeht. 
